Pacific

Journal of

Mathematics

ON THE METRIC STRUCTURE OF OPEN MANIFOLDS WITH NONNEGATIVE CURVATURE

LUis GUIJARRO

Volume $196 \quad$ No. 2

December 2000 


\title{
ON THE METRIC STRUCTURE OF OPEN MANIFOLDS WITH NONNEGATIVE CURVATURE
}

\author{
LuIS GuiJARRO
}

\begin{abstract}
An open manifold $M$ with nonnegative sectional curvature contains a compact totally geodesic submanifold $S$ called the soul. In his solution of the Cheeger-Gromoll conjecture, G. Perelman showed that the metric projection $\pi: M \rightarrow S$ was a $C^{1}$ Riemannian submersion which coincided with a map previously constructed by V. Sharafutdinov.

In this paper we improve Perelman's result to show that $\pi$ is in fact $C^{2}$, thus allowing us the use of O'Neill formulas in the study of $M$. For the proof, we study carefully how the conjugate locus of $S$ behaves in regard to the fibers of $\pi$. As applications, we study souls with totally geodesic Bieberbach submanifolds, and also obtain some rigidity results concerning the distribution of the rays of $M$.
\end{abstract}

\section{Introduction and main results.}

The structure of a complete noncompact manifold with nonnegative sectional curvature is specially simple from a differentiable point of view. The celebrated soul theorem of Cheeger and Gromoll ([5]) establishes that there is always some compact and totally geodesic submanifold $S$ such that the whole manifold is diffeomorphic to the normal bundle of $S$. Such a submanifold is called a soul.

However, in spite of such a strong statement, several of the most important metric properties of $M$ are hardly understood. A breakthrough was the resolution of the soul conjecture by Perelman $([8])$. It is this result that has motivated much of the present paper. In particular, we show the following theorem:

Theorem 1.1. The Sharafutdinov map $\mathrm{sh}: M \rightarrow S$ is a $C^{2}$ Riemannian submersion.

The interesting part of this result resides in the possibility of using most of the structure developed for smooth Riemannian submersions in our study of the metric relations between the manifold and its soul. In particular, the existence of such a Riemannian submersion together with the curvature conditions sets important restrictions on many of the main geometric objects 
of $M$. For example, we show in Section 10 how rays are forced to stay in Sharafutdinov fibers as well as to be invariant under the horizontal diffeomorphisms between fibers defined by the Riemannian submersion. We also find some restrictions of the same type obtained in [7] for the existence of metrics with nonnegative curvature on vector bundles over souls containing totally geodesic flat submanifolds. The result also gives some evidence to the conjecture that any submetry between Riemannian manifolds with nonnegative curvature is $C^{\infty}[\mathbf{2}]$. Furthermore, in the process of the proof we also obtain some interesting information on the focal set of the soul and its relation to the fibers of the Sharafutdinov map.

The paper is divided as follows: Section 2 introduces some of the necessary background and notation for the rest of the paper. Section 3 extends the proof of the main theorem in [8] to some vertical vectors not placed at the soul. Section 4 examines the focal set to the soul and its structure with respect to sh. Section 5 studies the possible nonsmooth directions for the Sharafutdinov fibers, and Section 6 shows how they are related under horizontal diffeomorphisms, as well as constructing the differentials of these. Section 7 proves the continuity of such extensions, and Section 8 wraps up the previous material to give a proof of Theorem 1.1. Finally the last two sections deal with the mentioned applications of the main result of this paper.

The author would like to thank Karsten Grove for continuous encouragement during the elaboration of the present work, Valeri Berestovskii for letting him know about his work in submetries, and Vitali Kapovitch for showing us the proof of Lemma 5.3.

\section{Some background and notation.}

In what follows, we will reserve $M$ for an open manifold with nonnegative curvature, $S$ for its soul and $p$ for a general point in $M$. By the Soul Theorem, $M$ is diffeomorphic to the normal bundle of $S$ in $M$, which will be denoted as $\nu(S)$. In [10], Sharafutdinov constructed a distance nonincreasing retraction sh : $M \rightarrow S$ that was rediscovered later in [13]. We will use $\bar{p}$ and $\bar{\alpha}$ instead of $\operatorname{sh}(p)$ and sh $\circ \alpha$ to simplify notation. This map was the key tool used by Perelman to prove the following fact:

Theorem $2.1([8])$.

(1) Let $\bar{\alpha}:[0, \infty] \rightarrow S$ be a geodesic with $\bar{\alpha}(0)=\bar{p}$, and $u \in \nu_{\bar{p}}(S)$ a normal vector to the soul. Let $U$ be the parallel transport of $U$ along $\bar{\alpha}$. Then

$$
R(t, s)=\exp _{\bar{\alpha}(t)} s U(t) \quad t \in[0, a], s \in[0, \infty)
$$

is a flat rectangle totally geodesically immersed in $M$.

(2) $\operatorname{sh}\left(\exp _{\bar{p}} t u\right)=\bar{p} \quad$ for all $t \in[0, \infty)$ 
(3) $\operatorname{sh}: M \rightarrow S$ is a $C^{1}$ Riemannian submersion.

It is trivial to check that Riemannian submersions send balls of radius $\mathrm{R}$ to balls of the same radius. This property can be used in the metric category to define submetries between metric spaces (see [1] for the necessary definitions). In [2], we proved that submetries between Riemannian manifolds are always $C^{1,1}$ Riemannian submersions, thus improving the regularity of the Sharafutdinov map a little. Our proof employs this fact at several stages.

We will use $F_{\bar{p}}$ to denote the Sharafutdinov fiber over a point $\bar{p}$ in the soul. $\mathcal{H}$ and $\mathcal{V}$ will denote respectively the horizontal and the vertical distributions associated to sh (see [3]).

Given $\bar{\alpha}:[a, b] \rightarrow S$ a geodesic in the soul, there is always a Lipschitz homeomorphism $h^{b}: F_{\bar{\alpha}(a)} \rightarrow F_{\bar{\alpha}(b)}$ obtained by lifting $\bar{\alpha}$ horizontally to each point of $F_{\bar{\alpha}(a)}$. We usually denote by $\alpha$ one of such lifts. $h^{b}$ is called a horizontal map. It is differentiable at points where the Sharafutdinov map is smooth, and its differential at such points can be obtained as follows: For $c:(-\varepsilon, \varepsilon) \rightarrow F_{\bar{\alpha}(a)}$ take the geodesic variation given by $V(t, s)=h^{t}(c(s))$. Then $d h^{t}\left(c^{\prime}(0)\right)=\partial_{s} V(t, 0)$. The Jacobi field along $\alpha$ associated to this geodesic variation is called a holonomy Jacobi field (see [3]).

We will also use $P_{b}^{\alpha}$ for the parallel transport of vectors along a curve $\alpha$ from $\alpha(a)$ to $\alpha(b)$, and finally, we will reserve $\gamma$ for geodesics starting at the soul and normal to it.

\section{Geodesic directions in the Sharafutdinov fibers.}

The existence of a vector that remains vertical under parallel transport along any horizontal geodesic puts important restrictions in the geometry of $M$. Namely, we can adapt Perelman's proof for his rigidity statement and obtain the following theorem:

Theorem 3.1. Let $v \in \mathcal{V}_{p}$ be a vertical vector that stays vertical under parallel transport along any horizontal piecewise broken geodesic. If $\alpha: \mathbb{R} \rightarrow$ $M$ is any horizontal geodesic with $\alpha(0)=p$ and $V(t)$ is the vector field along $\alpha$ obtained by the parallel transport of $v$, then

(1) $\operatorname{sh}(\exp s V(t))=\operatorname{sh}(\alpha(t))$ for all $t, s$.

(2) The rectangle

$$
W(t, s)=\exp _{\alpha(t)} s V(t)
$$

is flat and totally geodesically immersed in $M$.

(3) $h^{t}(W(0, s))=W(t, s)$ for $h^{t}$ the horizontal homeomorphism associated to $\alpha$.

(4) $\partial_{t} W(0, s)$ is the horizontal lift of $(\operatorname{sh} \circ \alpha)^{\prime}$ to $W(0, s)$.

Proof. Let $\mathcal{P}$ be the closure of the set of vectors in $T M$ that can be obtained from $v$ by parallel transport along piecewise smooth horizontal geodesics. 
Since horizontal curves remain at a constant distance from the soul, $\mathcal{P}$ is a compact set formed entirely by vertical vectors.

Next define a real function $f:[0, \infty) \rightarrow \mathbb{R}$ as

$$
f(s)=\max _{u \in \mathcal{P}} d\left(\operatorname{sh} \circ \sigma_{u}(s), \operatorname{sh} \circ \sigma_{u}(0)\right)
$$

where $\sigma_{u}$ is the geodesic tangent to $u$ at 0 .

The proof of the main theorem in [8] can be now reproduced verbatim to show that $f \equiv 0$, thus implying (1). The only minor modification is that instead of working with $\nu(S)$, we need to use $\mathcal{P}$ and horizontal geodesics through $p$. (2) is an easy application of Berger's rigidity lemma together with (1).

For (3), use that for $u^{*}=P_{t}^{\alpha} u$,

$$
d\left(\operatorname{sh} \circ \sigma_{u}(s), \operatorname{sh} \circ \sigma_{u^{*}}(s)\right)=d(\bar{\alpha}(0), \bar{\alpha}(t))=t
$$

and that $W(t, s)$ is a geodesic to conclude that it has to be exactly the lift of sh $\circ \alpha$ to $W(0, s)$. Finally (4) is a trivial consequence of (3) and the uniqueness of horizontal lifts.

In view of this, it is natural to ask to what vectors we can apply Theorem 3.1. Some examples are provided in the following corollary:

Corollary 3.2. The Sharafutdinov vector field and the perpendicular space to the interior of a totally convex set in the Cheeger-Gromoll exhaustion remain vertical under parallel transport along a horizontal geodesic.

Proof. The part about the Sharafutdinov vector field follows from Theorem A.5 in the appendix of [14], together with the fact that horizontal geodesics are entirely contained in the boundaries of the sets in the Cheeger-Gromoll exhaustion.

For the second part, let $C$ be one of the sets of the Cheeger-Gromoll exhaustion. By the above comment, horizontal geodesics at points of $C$ are entirely contained in $C$, and thus $\mathcal{H}$ is tangent to $C$. It is also wellknown that $C$ has the structure of a totally geodesic embedded submanifold $N$ with maybe nonsmooth boundary. So let $p \in C$ be a point that is not in its boundary, and decompose $T_{p} M$ as $T_{p} N \oplus T_{p} N^{\perp}$. Since $\mathcal{H} \subset T_{p} N$, $T_{p} N^{\perp} \subset \mathcal{V}$. If $\gamma$ is a horizontal geodesic, parallel transport along it will preserve $T_{p} N$ because of the totally geodesic condition, as well as its normal subspace. Thus elements of $T_{p} N^{\perp}$ remain vertical under horizontal parallel transport and the result follows.

\section{The focal set of the soul.}

In this section we include several simple consequences of Theorem 2.1.

Lemma 4.1. sh : $M \longrightarrow S$ is $C^{\infty}$ almost everywhere. 
Proof. Let $\exp : \nu(S) \rightarrow M$ be the normal exponential map of the soul $S$ in $M$, and $\pi: \nu(S) \rightarrow S$ the bundle projection. Then the following diagram commutes:

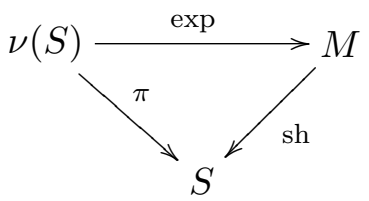

Thus sh $=\pi \circ \exp ^{-1}$ in any point where the exponential has a local inverse, namely, away from the set of focal points of $S$. By Sard's theorem, we can conclude that sh is smooth almost everywhere.

Thus the horizontal and vertical distributions $\mathcal{H}$ and $\mathcal{V}$ are also smooth almost everywhere. The only possible bad points are those in the focal set of $S$. However, the structure of these is intimately related to the Sharafutdinov fibers:

Lemma 4.2. If $p$ is a focal point of $S$ along $\gamma$, then $p$ is conjugate to $\operatorname{sh}(p)$ along $\gamma$. Furthermore, the focal set is invariant under horizontal homeomorphisms; i.e, if $\bar{\alpha}$ is a geodesic in $S$ and $h^{t}$ is its associated horizontal homeomorphism, then $h^{t}(p)$ is conjugate to its Sharafutdinov image along $h^{t}(\gamma)$.

Proof. Let $X(t)$ be the horizontal lift to $\gamma$ of some vector tangent to the soul at $\gamma(0)$. Because of Theorem 2.1, $X$ is a parallel Jacobi field. Hence, if $J$ is any other Jacobi field along $\gamma$,

$$
\langle J, X\rangle^{\prime \prime}=\left\langle J^{\prime \prime}, X\right\rangle=\left\langle R\left(J, \sigma^{\prime}\right) \sigma^{\prime}, X\right\rangle=\left\langle J, R\left(X, \sigma^{\prime}\right) \sigma^{\prime}\right\rangle=0 .
$$

Hence, if $J(0)$ is tangent to the soul with $J^{\prime}(0)$ vertical and $J(1)=0$, we conclude that $J(0)=0$, which proves the first part of the lemma.

For the second part, let

$$
u(\tau)=\gamma^{\prime}(0)+\tau J^{\prime}(0) \quad V(s, \tau)=\exp _{\bar{p}}(s u(\tau)) .
$$

By Theorem 2.1,

$$
V^{t}(s, \tau)=h^{t}(V(s, \tau))
$$

is a smooth geodesic variation of $h^{t} \gamma$. Thus $c_{1}(\tau)=V(1, \tau)$ and $c_{2}(\tau)=$ $V^{t}(1, \tau)$ are both differentiable curves. Since $h^{t}$ is Lipschitz $([2])$ and $c_{1}^{\prime}(0)=$ 0 , it is easy to see that $c_{2}^{\prime}(0)=0$. Thus if $Y$ is the Jacobi field associated to $V^{t}$, we obtain that $Y(0)=Y(1)=0$, while $Y^{\prime}(0)=P^{t} J^{\prime}(0) \neq 0$, which finishes the proof.

Using the above lemma, we can get a more refined statement about the measure of the focal set of $S$ : 
Lemma 4.3. Let $\Omega$ be the set of focal points of $S$. Then $\Omega \cap \operatorname{sh}^{-1}(\bar{p})$ has measure zero in $\operatorname{sh}^{-1}(\bar{p})$ for any $\bar{p} \in S$.

Proof. If for some $\operatorname{sh}^{-1}(\bar{p})$ its intersection with $\Omega$ were a set of positive measure in the fiber, the previous lemma would imply that the same should also be true for any other fiber. Applying Fubini's theorem, we would get that $|\Omega| \neq 0$, which is a contradiction.

\section{Conjugate Directions at Focal points.}

For $p \in M$, let $\gamma:[0,1] \rightarrow M$ be a geodesic normal to the soul joining $\bar{p}$ to $p$. Consider the following subspace of the set of vertical vectors at $p$ :

$$
\mathcal{V}_{\gamma}=\left(d \exp _{\bar{p}}\right)_{u}\left(\nu_{\bar{p}}(S)\right) \quad \text { where } u=\gamma^{\prime}(0) \text {. }
$$

This is the set of all vectors in $\mathcal{V}_{p}$ that can be written as $J(1)$, where $J$ is a Jacobi field along $\gamma$ with $J(0)=0$ and $J^{\prime}(0) \in \nu_{\bar{p}}(S)$.

Definition 5.1. The vertical vectors in $\mathcal{V}_{\gamma}^{\perp}$ are called conjugate directions for $\gamma$. Its union will be denoted by $\mathcal{C}_{\gamma}$.

If $\mathcal{V}_{p}=\mathcal{V}_{\gamma}$ for some geodesic $\gamma, p$ is not focal for $S$ along $\gamma$, and consequently it will be a smooth point for sh because of diagram (4.1). Otherwise, we need to consider the following lemmas:

Lemma 5.2. If $J$ be a Jacobi field along $\gamma$ with $J(0)=J(1)=0$, then $J$ and $J^{\prime}$ are vertical.

Proof. If $X$ is the horizontal lift to $\gamma$ of some vector in $T_{\bar{p}} S$, then

$$
\langle X, J\rangle^{\prime \prime}=\left\langle X, J^{\prime \prime}\right\rangle=\langle X, R(J, T) T\rangle=\langle R(X, T) T, J\rangle=0 .
$$

Hence $\langle X, J\rangle$ is a linear function vanishing twice. This ends the proof since $X$ is arbitrary.

Lemma 5.3. Let $v \in \mathcal{C}_{\gamma}$. Then there is a vertical Jacobi field $J$ along $\gamma$ with $J(0)=0, J(1)=0$ and $J^{\prime}(1)=v$.

Proof. Define $J$ by the last two conditions. We know that for any other Jacobi field $Y$ along $\gamma$,

$$
\left\langle J, Y^{\prime}\right\rangle-\left\langle J^{\prime}, Y\right\rangle=a
$$

for some constant $a$. If $Y$ satisfies $Y(0)=0$ and $Y^{\prime}(0) \in \nu(S)$, then $a=0$ since $J(1)=0$ and $J^{\prime}(1) \in \mathcal{V}_{\gamma}^{\perp}$.

At $t=0$,

$$
\left\langle J(0), Y^{\prime}(0)\right\rangle-\left\langle J^{\prime}(0), Y(0)\right\rangle=0 .
$$

But $Y(0)=0$, and since $Y^{\prime}(0)$ is arbitrary, it follows that $J(0)=0$.

Lemma 5.4. Let $\alpha: \mathbb{R} \rightarrow M$ be a piecewise smooth horizontal geodesic passing through $p$. For any vector $v \in \mathcal{C}_{\gamma}$, the parallel transport of $v$ along $\alpha$ remains vertical. 
Proof. Let $J$ be the Jacobi field along $\gamma$ with $J^{\prime}(1)=v$ given by Lemma 5.3. One associated geodesic variation is:

$$
V(s, \tau)=\exp _{\bar{p}}\left(s \gamma^{\prime}(0)+s \tau J^{\prime}(0)\right) .
$$

Let $h^{T}: F_{\alpha(0)} \rightarrow F_{\alpha(T)}$ be the horizontal homeomorphism induced by $\alpha$. Due to Theorem 2.1, $h^{T} V(s, \tau)$ is another geodesic variation whose Jacobi field $J^{T}$ vanishes for $s=0$ and $s=1$. By Lemma 5.2, $J^{T}$ and $\left(J^{T}\right)^{\prime}$ are vertical.

Consider next the parametrized surface $f: \mathbb{R} \times(-\epsilon, \epsilon) \rightarrow M$ defined as

$$
f(T, \tau)=h^{T} V(1, \tau) .
$$

Clearly $T \rightarrow f(T, \tau)$ is a horizontal geodesic for each $\tau$, and because of the proof of Lemma $4.2, \partial_{\tau} f(T, 0)=0$. The vector field along $f$ defined by

$$
W(T, \tau)=\left(\partial_{s} h^{T} V\right)(1, \tau)
$$

is tangent to geodesics connecting $f(T, \tau)$ to their Sharafutdinov images on $S$. Therefore, by Theorem 2.1,

$$
W(T, \tau)=P_{T} W(0, \tau)
$$

where now we are using $P_{T}$ for the parallel transport of vectors along the geodesics $T \rightarrow f(T, \tau)$. Thus,

$$
0=\frac{D}{\partial \tau} \frac{D}{\partial T} W(T, \tau)=\frac{D}{\partial T} \frac{D}{\partial \tau} W(T, \tau)+R\left(\frac{\partial f}{\partial T}, \frac{\partial f}{\partial \tau}\right) W .
$$

For $\tau=0$, the second vector in the curvature term vanishes. Hence $\frac{D}{\partial \tau} W(T, 0)$ is the parallel transport of $v$ along $\alpha$. Since it is also $\left(J^{T}\right)^{\prime}(1)$ Lemma 5.2 implies that it is vertical.

Remark 5.5. The proof of this lemma also provides two facts that will be required later. The first one is that $P_{T}^{\alpha} v=\left(J^{T}\right)^{\prime}(1)$. The second is that if $J^{\prime}(0) \in \operatorname{ker} d \exp _{u}$, then $P_{T}^{\bar{\alpha}} J^{\prime}(0) \in \operatorname{ker} d \exp _{P_{T} u}$, or in other words, the kernel of $d \exp$ is invariant under parallel transport along curves contained in the soul.

\section{Holonomy Jacobi fields at the focal set.}

From now on and until the end of the proof, $p$ will denote a focal point for $S$. Let $\mathcal{R}_{p}$ be the set of vertical geodesics with $\gamma(0)=\bar{p}$ and $\gamma(1)=p$. For each $\gamma \in \mathcal{R}_{p}$ we have an orthogonal splitting $\mathcal{V}_{p}=\mathcal{V}_{\gamma} \oplus \mathcal{C}_{\gamma}$ into conjugate and non-conjugate directions along $\gamma$.

Define the subspace of $\mathcal{V}_{p}$ given by

$$
\mathcal{W}_{p}=\bigcap_{\gamma \in \mathcal{R}_{p}} \mathcal{V}_{\gamma} .
$$


Then $\mathcal{W}_{p}^{\perp}$ is the subspace generated by the union of the $\mathcal{C}_{\gamma}$, and by Lemma 5.4 any of its elements stays vertical under parallel transport along horizontal geodesics.

Remark 6.1. On the other hand, for any $a \in \mathcal{W}_{p}$ and $\gamma \in \mathcal{R}_{p}$, we can find a curve of the form $c(s)=\exp _{\bar{p}}(u(s))$ with $u(0)=\gamma^{\prime}(0)$ and $c^{\prime}(0)=a$. However, given an element in $\mathcal{W}_{p}^{\perp}$ this could be true for some of the geodesics in $\mathcal{R}_{p}$, but certainly not for all.

Recall that holonomy Jacobi fields are always defined for nonfocal points of the soul, since these are points where sh is smooth. We now define them also in focal points, and in Section 7 we will show that this extension is continuous. The motivation for this definition comes from the smooth case when $a \in \mathcal{W}_{p}$ and from Lemma 5.4 for $a \in \mathcal{W}_{p}^{\perp}$.

Definition 6.2. Let $\alpha: \mathbb{R} \rightarrow M$ a horizontal geodesic with initial point $p$ :

(1) For $a \in \mathcal{W}_{p}$, choose any $\gamma \in \mathcal{R}_{p}$ and a curve $u(s)$ formed by vectors normal to $S$ at $\bar{p}$ with $u(0)=\gamma^{\prime}(0)$ and $(d \exp )_{\gamma^{\prime}(0)}\left(u^{\prime}(0)\right)=a$. Define

$$
J(t)=\left.\frac{d}{d s}\right|_{s=0} \exp _{\bar{\alpha}(t)}\left(P_{t}^{\bar{\alpha}} u(s)\right) .
$$

(2) On the other hand, if $a \in \mathcal{W}_{p}^{\perp}$ define

$$
J(t)=P_{t}^{\alpha} a \text {. }
$$

We will call $J$ the pseudoholonomy Jacobi field along $\alpha$ corresponding to $a$.

The definition of $J(t)$ for $a \in \mathcal{W}_{p}$ is independent of the choices of $\gamma$ and $u(s)$ because of the following lemma:

Lemma 6.3. Let $a \in \mathcal{W}_{p}, \gamma_{1}, \gamma_{2} \in \mathcal{R}_{p}$ and $u_{1}(s), u_{2}(s)$ smooth curves in $\nu_{\bar{p}}(S)$ as in the first part of Definition 6.2. Then the curves $c_{i}(s)=$ $h^{t} \exp _{\bar{p}} u_{i}(s)$ for $i=1,2$ are smooth curves tangent to each other at $s=0$.

Proof. Smoothness is a trivial consequence of Theorem 2.1. In fact,

$$
h^{t} \exp _{\bar{p}} u_{i}(s)=\exp _{\bar{\alpha}(t)} P_{t}^{\bar{\alpha}} u_{i}(s) .
$$

Therefore, if $\left(y_{1}, \ldots, y_{n}\right)$ is a parametrization around $c_{1}(0)$, we have that for each coordinate function

$$
y_{j} \circ c_{i}(s)=y_{j} \circ c_{i}(0)+\left(y_{j} \circ c_{i}\right)^{\prime}(0) s+o(s) .
$$

Since $h^{t}$ is Lipschitz,

$$
\left|y_{j} \circ c_{1}(s)-y_{j} \circ c_{2}(s)\right| \leq K o(s) .
$$

Dividing by $s$ and letting it approach to zero, we get the required statement.

A similar argument shows: 
Lemma 6.4. Let $a \in \mathcal{W}_{p}^{\perp}$. Suppose there is a $\gamma \in \mathcal{R}_{p}$ with $a \in \mathcal{V}_{\gamma}$, and let $u(s)$ be a curve in $\nu_{\bar{p}}(S)$ as before. Define $c_{1}(s)=h^{t} \exp _{\bar{p}} u(s)$ and $c_{2}(s)=h^{t} \exp _{p}(s v)$. Then $c_{1}, c_{2}$ are smooth curves tangent to each other at $s=0$.

Proof. Smoothness of $h^{t} c_{2}(s)$ follows from Lemma 5.4. The rest of the proof mimics exactly that of 6.3 .

We can now extend the definition of $J(t)$ to an arbitrary vertical vector at $p$ by linearity. We will think of these fields as extending the differential of $h^{t}$ to the focal points of $S$. Along these lines, we can see the $J$ 's obtained from $\mathcal{W}_{p}, \mathcal{W}_{p}^{\perp}$ as "partial derivatives" for $h^{t}$. In the next section we will show that they are continuous, and from there it will be easy to obtain higher regularity for sh.

\section{Continuity of the holonomy Jacobi fields.}

We start this section with a simple technical lemma:

Lemma 7.1. Let $f: \mathbb{R}^{n} \rightarrow \mathbb{R}^{n}$ be a Lipschitz map which is also $C^{\infty}$ almost everywhere. For any compact set $K \in \mathbb{R}^{n}$, there is a constant $C$ so that for any $p \in K$ in the domain of smoothness of $f$, we have $\left\|d f_{p}(u)\right\| \leq C\|u\|$.

Proof. The proof is an easy application of Taylor's theorem to the coordinate functions of $f(c(t))$, where $c$ is some smooth curve in $\mathbb{R}^{n}$. In fact, we can take as $C$ any Lipschitz constant for $f$ in $K$.

In Section 6 we have constructed "holonomy" Jacobi fields at every point of $M$ along any horizontal geodesic given any vertical vector as initial condition. To prove the continuity of these, we will take a sequence of nonfocal points $\left\{p_{i}\right\}$ approaching $p \in M$, and $\left\{\gamma_{i}\right\} \subset \mathcal{R}_{p_{i}}$ approaching some $\gamma \in \mathcal{R}_{p}$. We also take sequences of vectors $\left\{x_{i}\right\} \in \mathcal{H}_{p_{i}},\left\{v_{i}\right\} \in \mathcal{V}_{p_{i}}$ with limits $x$ and $v$ respectively. For each pair $\left\{x_{i}, v_{i}\right\}$, let $J_{i}$ be the holonomy Jacobi field along the horizontal geodesic determined by $x_{i}$ with $J_{i}(0)=v_{i}$, and for $x, v$, define $J$ using Section 6 . The fact that the $\left\{p_{i}\right\}$ are nonfocal is not a serious restriction since this is a dense set in $M$.

Lemma 7.2. If $v \in \mathcal{W}_{p}$, then $J_{i}(t) \rightarrow J(t)$.

Proof. Take curves $u_{i}(s)$ in $\nu_{p_{i}}(S)$ as in Remark 6.1. We can always assume that $u_{i}^{\prime}(0) \rightarrow u^{\prime}(0)$ for some curve $u(s)$ in $\nu_{p}(S)$ : In fact, we could have just taken $u_{i}(s)=\gamma_{i}^{\prime}(0)+s \bar{a}_{i}$ for some $\bar{a}_{i} \in \nu_{\bar{p}_{i}}(S)$ and $u(s)=\gamma^{\prime}(0)+s \bar{a}$ for $\bar{a}$ some limit point of the $\bar{a}_{i}$. Such a limit exists because the $\bar{a}_{i}$ remain bounded.

Clearly

$$
v_{i}=d \exp \left(u_{i}^{\prime}(0)\right) \rightarrow d \exp \left(u^{\prime}(0)\right)
$$


and therefore $d \exp \left(u^{\prime}(0)\right)=v$. For a given $t$,

$$
J_{i}(t)=\left.\left.\frac{d}{d s}\right|_{s=0} \exp P_{t}^{\bar{\alpha}_{i}} u_{i}(s) \rightarrow \frac{d}{d s}\right|_{s=0} \exp P_{t}^{\bar{\alpha}} u(s)
$$

because $d \exp$ is continuous, $\bar{\alpha}_{i}$ approaches $\bar{\alpha}$ in $[0, t]$ and $u_{i}^{\prime}(0)$ approaches $u^{\prime}(0)$. Since the right hand side of $(7.1)$ is exactly $J(t)$, the lemma follows.

Next we will prove continuity for vectors in $\mathcal{W}_{p}^{\perp}$.

Lemma 7.3. If $v \in \mathcal{W}_{p}^{\perp}$, then $J_{i}(t) \rightarrow J(t)$.

Proof. By Lemma 7.1, all $J_{i}(t)$ are uniformly bounded in norm in any fixed interval $[0, T]$, and therefore there is some subsequence converging to a Jacobi field $\widetilde{J}(t)$ along $\alpha$ in $[0, T]$ with $\widetilde{J}(0)=v$. If $v \in \mathcal{V}_{\gamma}$, then $J=\widetilde{J}$ because of Lemmas 6.4 and the proof of 7.2.

Otherwise, suppose first that $v \in \mathcal{V}_{\gamma}^{\perp}$, and let $V(t, s)$ be a geodesic variation along $\gamma$ with $V(0, s)=\bar{p}$, and associated Jacobi field $Y$ satisfying $Y(1)=0$, and $Y^{\prime}(1)=v$; for example,

$$
V(r, s)=\exp _{\bar{p}}(r(\bar{u}+s \bar{w}))
$$

for some $\bar{w} \in \nu_{\bar{p}}(S)$. Choose some sequence of vectors $\bar{w}_{i} \in \nu_{\overline{p_{i}}}(S)$ with $\bar{w}_{i} \rightarrow \bar{w}$ and construct the geodesic variations

$$
V_{i}(r, s)=\exp _{\overline{p_{i}}}\left(r\left(\bar{u}_{i}+s \bar{w}_{i}\right)\right) .
$$

If $Y_{i}$ are their corresponding Jacobi fields, then $Y_{i}^{\prime}(1) \rightarrow Y^{\prime}(1)$ by continuity. Thus $Y_{i}^{\prime}(1)$ and $J_{i}(0)$ converge to the same limit, and for a fixed value of $t$,

$$
\left\|d h^{t}\left[Y_{i}^{\prime}(1)-J_{i}(0)\right]\right\| \leq C\left\|Y_{i}^{\prime}(1)-J_{i}(0)\right\| \rightarrow 0
$$

which means that since $d h^{t}\left(J_{i}(0)\right)=J_{i}(t)$, we just need to show that $d h^{t}\left[Y_{i}^{\prime}(1)\right] \rightarrow J(t)$.

Along $V_{i}$ and $V$, we have an explicit expression for $h^{t}$; namely,

$$
h^{t} \circ V_{i}(r, s)=\exp _{\bar{\alpha}_{i}(t)}\left(P_{t}^{\bar{\alpha}_{i}}\left[r\left(\bar{u}_{i}+s \bar{w}_{i}\right)\right]\right)
$$

with a similar expression for $h^{t} \circ V$. These are again geodesic variations whose Jacobi fields along $h^{t} \circ \gamma_{i}$ and $h^{t} \circ \gamma$ we denote as $\widetilde{Y}_{i}$ and $\widetilde{Y}$ respectively. From (7.2), it follows trivially that $h^{t} \circ V_{i}, \widetilde{Y}_{i}, \widetilde{Y}_{i}^{\prime}$ converge to $h^{t} \circ V, \widetilde{Y}$ and $\widetilde{Y}^{\prime}$ respectively. Furthermore, $\widetilde{Y}_{i}=d h^{t}\left(Y_{i}\right)$ and $\widetilde{Y}=d h^{t}(Y)$.

We will prove next that $\widetilde{Y}_{i}^{\prime}(1)$ approaches the same limit as the sequence formed by the $d h^{t}\left(Y_{i}^{\prime}(1)\right)$. This will conclude the proof, since as previously mentioned,

$$
\lim \tilde{Y}_{i}^{\prime}(1)=\tilde{Y}^{\prime}(1)=J(t)
$$

where the second identity comes from Remark 5.5. 
In order to do this, write $Y_{i}(r)$ as $f_{i}(r) E_{i}(r)$ where $f_{i}$ is some function with $f_{i}(0)=0, f_{i}^{\prime}(0) \neq 0$, and $E_{i}$ is some vector field along $\gamma$ with $E_{i}(1) \neq 0$. Clearly $f_{i}(1) \rightarrow 0$, and thus for $\widetilde{Y}_{i}(r)=d h^{t}\left(Y_{i}(r)\right)=f_{i}(r) d h^{t}\left(E_{i}(r)\right)$ we have that

$$
\begin{aligned}
\widetilde{Y}_{i}^{\prime}(1) & =f_{i}^{\prime}(1) d h^{t}\left(E_{i}(1)\right)+f_{i}(1) d h^{t}\left(E_{i}\right)^{\prime}(1) \rightarrow \lim f_{i}^{\prime}(1) d h^{t}\left(E_{i}(1)\right) \\
& =\lim d h^{t}\left(Y_{i}^{\prime}(1)\right)
\end{aligned}
$$

as we wanted to show.

Finally, if $v \notin \mathcal{V}_{\gamma}^{\perp}$, let $v=v_{1}+v_{2}$ with $w_{1} \in \mathcal{V}_{\gamma}$ and $w_{2} \in \mathcal{V}_{\gamma}^{\perp}$. Clearly, we can decompose the sequence of vertical vectors $\left\{v_{i}\right\}$ as a sum of sequences, one of which is approaching $w_{1}$ and the other $w_{2}$. Then the lemma follows from an application of the previous two cases.

\section{Proof of the Main Theorem.}

Recall that for a Riemannian submersion, the O'Neill tensors and the holonomy Jacobi fields are related by the equation

$$
J^{\prime}(0)=J^{\prime}(0)^{H}+J^{\prime}(0)^{V}=A_{x} v+T_{v} x
$$

where $J(t)$ is supposed to be the holonomy Jacobi field along the horizontal geodesic determined by $x$ with initial condition $v$.

In the case of the Sharafutdinov map, Equation (8.1) can be used to define extensions of the O'Neill tensors to the focal points of the soul. We will call such extensions $\bar{A}$ and $\bar{T}$.

Lemma 8.1. $\bar{A}$ and $\bar{T}$ are continuous.

Proof. We just need to check that if $E$ and $F$ are smooth vector fields, then $\bar{A}_{E^{H}} F^{V}$ and $\bar{T}_{F^{V}} E^{H}$ are continuous. So assume we have some sequence $p_{i} \rightarrow p$. For $J_{i}(t), J(t)$ the holonomy Jacobi fields corresponding to $F^{V}\left(p_{i}\right), E^{H}\left(p_{i}\right)$ and $F^{V}(p), E^{H}(p)$, we know already that $J_{i}(t) \rightarrow J(t)$. Thus $J_{i}^{\prime}(0) \rightarrow J^{\prime}(0)$, and hence

$$
\begin{aligned}
J_{i}^{\prime}(0)^{H} & =\bar{A}_{E^{H}} F^{V}\left(p_{i}\right) \rightarrow J^{\prime}(0)^{H}=\bar{A}_{E^{H}} F^{V}(p) \\
J_{i}^{\prime}(0)^{V}=\bar{T}_{F^{V}} E^{H}\left(p_{i}\right) & \rightarrow J^{\prime}(0)^{V}=\bar{T}_{F^{V}} E^{H}(p) .
\end{aligned}
$$

Lemma 8.2. $\mathcal{H}$ and $\mathcal{V}$ are $C^{1}$

Proof. If $p \in M$ is a point where sh is smooth, then

$$
\left(\nabla_{E} F\right)^{H}=\left(\nabla_{E} F^{H}\right)^{H}+\bar{A}_{E^{H}} F^{V}+\bar{T}_{E^{V}} F^{V}
$$

for any smooth vector fields $E$ and $F$. Therefore,

$$
\left(\nabla_{E} F^{H}\right)^{H}=\left(\nabla_{E} F\right)^{H}-\bar{A}_{E^{H}} F^{V}-T_{E^{V}} F^{V} \text {. }
$$


Analogously, we also get

$$
\begin{aligned}
& \left(\nabla_{E} F^{H}\right)^{V}=\bar{A}_{E^{H}} F^{H}+\bar{T}_{E^{V}}^{*} F^{H} \\
& \left(\nabla_{E} F^{V}\right)^{H}=\bar{A}_{E^{H}}^{*} F^{V}+\bar{T}_{E^{V}} F^{V}
\end{aligned}
$$

and

$$
\left(\nabla_{E} F^{V}\right)^{V}=\left(\nabla_{E} F\right)^{V}-\bar{A}_{E^{H}} F^{H}-\bar{T}_{E^{V}} F^{H} .
$$

Clearly, the right hand side of these equations can be extended to continuous vector fields all over $M$.

For $p \in M$, let $\phi: U \rightarrow \mathbb{R}^{n}, \phi=\left(x_{1}, \ldots, x_{n}\right)$, be a coordinate system centered at $p$, and let $H, V \subset T\left(\mathbb{R}^{n}\right)$ be defined by $H=\phi_{*}(\mathcal{H}), V=\phi_{*}(\mathcal{V})$. It is clear that $H$ and $V$ are Lipschitz on $\phi(U)$ and smooth for almost every point where they are defined.

For a smooth vector field $E$ around $p$, let $\widetilde{E}=\phi_{*}(E)$ be its image in $T\left(\mathbb{R}^{n}\right)$. If $W=\left(W_{1}, \ldots, W_{n}\right)$ is its $H$-component, then

$$
\nabla_{\partial_{i}}\left(\sum_{j} W_{j} \partial_{j}\right)=\sum_{k}\left\{\frac{\partial W_{k}}{\partial x_{i}}+\sum_{j} \Gamma_{i j}^{k} W_{j}\right\} \partial_{k} .
$$

The left hand side can be extended to a continuous vector field by the previous discussion. This implies that each $W_{k}$ is a Lipschitz real valued function which is almost everywhere smooth and whose partial derivatives admit a continuous extension everywhere. Therefore, since $W_{k}$ is absolutely continuous, we can recover it from its derivatives just by integration. Consequently, $W_{k}$ is differentiable everywhere, and its derivatives have the type of regularity given by (8.2), i.e., they are continuous. Hence $H$ and $\mathcal{H}$ are $C^{1}$. Since $\mathcal{V}$ is just the orthonormal complement of $\mathcal{H}$, it inherits the same regularity of $\mathcal{H}$, thus finishing the proof of the lemma.

The main Theorem follows now because a submersion with $C^{r}$ fibers is itself $C^{r}$, as can be checked easily using coordinate charts adapted to the submersion.

Remark 8.3. The question of how much we can improve Theorem 1.1 is still open. At this moment, it is unclear to the author how the arguments from the previous sections could be rewritten to get higher regularity. However, thanks to the arguments of [6], it can be shown that if $M$ is an open manifold with nonnegative curvature, then we can always construct another nonnegatively curved metric for which the corresponding Sharafutdinov map is a $C^{\infty}$ Riemannian submersion. This should be useful when trying to prove nonexistence of metrics with nonnegative curvature over certain vector bundles. 


\section{Vector bundles of nonnegative curvature over partly flat souls.}

The problem of what vector bundles over compact manifolds have metrics of nonnegative curvature is still far from being solved. The case in which the soul is a Bieberbach manifold has been studied in [7], where it is proved that such bundles have to admit flat connections. This sets some restrictions in the topological properties of such bundles.

As an easy application of our main theorem, we can get a mild generalization of such a result when the metric in the soul has a totally geodesically embedded Bieberbach submanifold. We call such metrics partly flat.

Let $(S, g)$ be a compact manifold with a metric of non negative curvature, and suppose there is a totally geodesic embedding of a Bieberbach manifold

$$
i: B^{\prime} \rightarrow S
$$

with image $i\left(B^{\prime}\right)=B \subseteq S$. Let $M$ be an open manifold with nonnegative curvature and soul isometric to $(S, g)$. As usual, we denote by $\nu(S)$ the normal bundle of $S$ in $M$. Let $E=i^{*} \nu(S)$ be the pullback bundle of $\nu(S)$ by $i$.

Proposition 9.1. $e(E)=0$, where $e(E)$ is the integral Euler class of the bundle E.

Proof. Since $i: B^{\prime} \rightarrow B$ is a diffeomorphism, it is clear that $E$ is equivalent to the restriction of $\nu(S)$ over $B$. Call such bundle $F$. Let $\widetilde{\mathcal{H}}$ be the connection on $F$ obtained by normal parallel transport in $B$. We will show next that $\widetilde{\mathcal{H}}$ is flat. For this, let $\bar{x}, \bar{y}$ be vectors tangent to $B$ at some point $\bar{p}$. Take some $u \in \nu_{\bar{p}}(S)$ and $J(t)$ the Jacobi field along $\gamma_{u}(t)=\exp _{\bar{p}}(t u)$ with initial conditions $J(0)=0, J^{\prime}(0)=-\frac{1}{2} R^{\perp}(\bar{x}, \bar{y}) u$, where $R$ is the curvature tensor of $F$ with the mentioned connection.

We can use the proof of Proposition 1.7 in [12] to see that $J(t)=$ $A_{X} Y\left(\gamma_{u}(t)\right)$, where $X, Y$ are the horizontal lifts of $\bar{x}, \bar{y}$ to $\gamma_{u}(t)$. O'Neill's formula for the horizontal curvatures holds for a $C^{2}$ Riemannian submersion, and therefore

$$
0=K(\bar{x}, \bar{y})=K(x, y)+3\left\|A_{X} Y\right\|^{2} .
$$

The nonnegativity of $K(x, y)$ implies that $A_{X} Y=0$. Hence $J(t)=0$ and thus $R^{\perp}(\bar{x}, \bar{y}) u=0$, making $F$ flat.

As stated in [7], the integral Euler class of any flat vector bundle vanishes ([9]). This concludes the proof of the theorem.

\section{Restrictions on rays.}

The non negativity of the sectional curvature sets important restrictions on the behavior of rays. In this section, we will show that they lie on Sharafutdinov fibers and are invariant under horizontal diffeomorphisms. We will use the following theorem from Cheeger and Gromoll. 
Theorem 10.1 ([4], Thm. 8.22). Let $M$ be a complete manifold with non negative sectional curvature. whose Cheeger-Gromoll exhaustion function is denoted as $\left\{C^{a}\right\}_{a>0}$, with $C^{a} \supset C_{0} \supset \cdots \supset C_{k}=S$ as described in [4].

(1) If $\tau:(\infty, \infty) \rightarrow M$ is a geodesic and $\tau \subset C$ for some compact set $C$, then $\tau \subset \partial C^{a}$ for some $a>0$, or $\gamma \subset C_{0}$.

(2) If $\sigma, \tau:[0, \infty) \rightarrow M$ are geodesics such that

$$
\sigma(0)=\tau(0) \quad\left\langle\sigma^{\prime}(0), \tau^{\prime}(0)\right\rangle>0
$$

and $\sigma$ is a ray, then $\tau$ goes to infinity.

(3) Let $\sigma, \tau$ be as in (2) except $\left\langle\sigma^{\prime}(0), \tau^{\prime}(0)\right\rangle=0$. Suppose that $\tau$ does not go to infinity. If $V$ denotes the parallel vector field along $\tau$ generated by $\sigma^{\prime}(0)$, then

$$
W:[0, \infty) \times[0, \infty) \rightarrow \exp _{\tau(t)} s V(t)
$$

defines a flat (immersed) totally geodesic rectangle.

Note. The version of the Theorem that appears in [4] states some other facts that we have omitted here since they will not be needed.

Observe that any horizontal geodesic remains at a constant distance from the soul, and therefore never goes to infinity.

Lemma 10.2. Let $\sigma:[0, \infty) \rightarrow M$ be a ray. Then $\sigma$ is entirely contained in the Sharafutdinov fiber passing through $\sigma(0)$.

Proof. If $\tau:(-\infty, \infty) \rightarrow M$ is a horizontal geodesic with $\tau(0)=\sigma(t)$, it follows from the observation right before this lemma that $\tau$ does not go to infinity on either side. From Theorem 10.1, (2), we obtain

$$
\left\langle\sigma^{\prime}(t), \tau^{\prime}(0)\right\rangle \leq 0 \quad \text { and } \quad\left\langle\sigma^{\prime}(t),-\tau^{\prime}(0)\right\rangle \leq 0 .
$$

So $\sigma^{\prime}(t)$ is orthogonal to $\tau^{\prime}(0)$, and consequently, $\sigma^{\prime}(t)$ is vertical. Since $t$ was arbitrary,

$$
(\operatorname{sh} \circ \sigma)^{\prime}(t)=\operatorname{sh}_{*}\left(\sigma^{\prime}(t)\right)=0
$$

for all $t$. Therefore, $\operatorname{sh} \circ \sigma$ is constant, and the result follows.

Lemma 10.3. Let $\sigma:[0, \infty) \rightarrow M$ be a ray and $\tau:(-\infty, \infty) \rightarrow M a$ horizontal geodesic with $\sigma(0)=\tau(0)$. Denote by $V(t)$ the parallel vector field along $\tau(t)$ generated by $\sigma^{\prime}(0)$. Then

$$
W:(-\infty, \infty) \times[0, \infty) \longrightarrow M
$$

defined as

$$
W(t, s)=\exp _{\tau(t)} s V(t)
$$

is a flat immersed totally geodesic rectangle.

Proof. This is just part (3) from Theorem 10.1, together with the facts that the tangent vector to a ray is vertical, and horizontal geodesics remain in compact sets. 
Lemma 10.4. With the notation of Lemma $10.3, W\left(t_{0}, s\right)$ is a ray for $s \in$ $[0, \infty)$ and $W\left(t, s_{0}\right)$ are horizontal geodesics.

Proof. Let $b_{\sigma}$ be the Buseman function corresponding to $\sigma$. By the above lemma, $\alpha(t)=W\left(t, s_{0}\right)$ is a geodesic that stays in a compact set. Therefore $b_{\sigma}$ is constant over $\alpha$ and $b_{\sigma}\left(W\left(t_{0}, s\right)\right)=b_{\sigma}(\sigma(t))=t$, which means that $W\left(t, s_{0}\right)$ is a ray asymptotic to $\sigma([\mathbf{1 1}])$.

Thanks to Lemma 10.2, $W\left(t, s_{0}\right)$ is entirely contained in the Sharafutdinov fiber through $W\left(0, s_{0}\right)$. Since the lengths of the curves $W\left(t, s_{0}\right)$ are the same as that of $W(t, 0)$ when $t$ ranges in the same interval, and horizontal lifts of geodesics are unique, the last part of the lemma follows.

Corollary 10.5. Horizontal diffeomorphisms take rays to rays.

Therefore, the ray configuration is the same in every fiber.

\section{References}

[1] V.N. Berestovskii, Submetries of space forms of negative curvature, Siberian Mathematical Journal, 28 (1987), 552-562.

[2] V. Berestovskii and L. Guijarro, A metric characterization of Riemannian submersions, preprint.

[3] A.L. Besse, Einstein Manifolds, Vol. 10 of Ergebnisse der Mathematik und ihrer Grenzgebiete; 3, Springer Verlag, 1987.

[4] J. Cheeger and D.G. Ebin, Comparison Theorems in Riemannian Geometry, Vol. 9 of North Holland Mathematical Library, North Holland Publishing Company, 1975.

[5] J. Cheeger and D. Gromoll, On the structure of complete manifolds of nonnegative curvature, Annals of Mathematics, 96 (1972), 413-443.

[6] L. Guijarro, Improving the metric in an open manifold with nonnegative curvature, Proceedings of the AMS, 126 (1998), 1541-1545.

[7] M. Ozaydin and G. Walschap, Vector bundles with no soul, Proceedings of the American Mathematical Society, 120 (1994), 565-567.

[8] G. Perelman, Proof of the soul conjecture of Cheeger and Gromoll, Journal of Differential Geometry, 40 (1994), 209-212.

[9] W.A. Poor, Differential Geometric Structures, McGraw-Hill Book Company, 1981.

[10] V.A. Sharafutdinov, The Pogorelov-Klingenberg theorem for manifolds homeomorphic to $\mathbb{R}^{n}$, Siberian Mathematical Journal, 18 (1977), 915-925.

[11] K. Shiohama, Topology of complete noncompact manifolds, in 'Geometry of Geodesics and Related Topics', Vol. 3 of Advanced Studies in Pure Mathematics, (1984), 423450 .

[12] M. Strake and G. Walschap, $\Sigma$-flat manifolds and Riemannian submersions, Manuscripta Mathematica, 64 (1989), 213-226.

[13] J.-W. Yim, Distance nonincreasing retraction on a complete open manifold of nonnegative sectional curvature, Ann. Global Anal. Geom., 6 (1988), 191-206. 
[14] _ Space of souls in a complete open manifold of nonnegative curvature, Journal of Differential Geometry, 32 (1990), 429-455.

Received March 8, 1999.

University of Pennsylvania

Philadelphia, PA 19104

E-mail address: guijarro@math.upenn.edu

Universidad Autónoma de Madrid SPAIN 